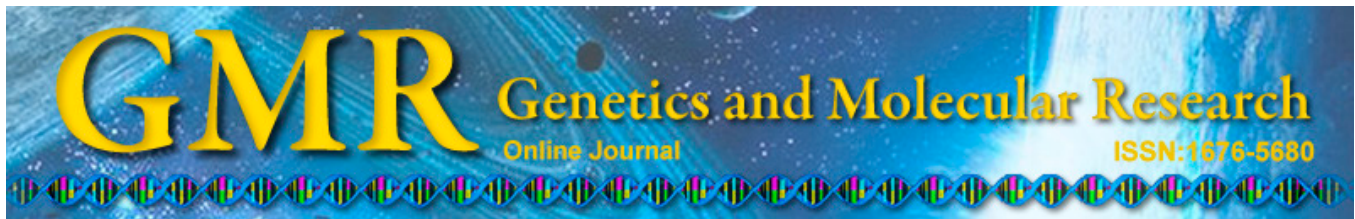

\title{
Mutation analysis and prenatal diagnosis for three families affected by isolated methylmalonic aciduria
}

\author{
X.D. Kong', H.R. Shi ${ }^{1}$, N. Liu ${ }^{1}$, Q.H. Wu ${ }^{1}$, X.J. Xu ${ }^{2}$, Z.H. Zhao ${ }^{1}$, N. Lu ${ }^{1}$, \\ J. Li-Ling ${ }^{3}$ and D. Luo ${ }^{4}$ \\ ${ }^{1}$ Center of Prenatal Diagnosis, \\ The First Affiliated Hospital of Zhengzhou University, Zhengzhou, Henan, China \\ ${ }^{2}$ Department of Pediatrics, The First Affiliated Hospital of Zhengzhou University, \\ Zhengzhou, Henan, China \\ ${ }^{3}$ Sino-Dutch Biomedical and Information Engineering School, \\ Northeastern University, Shenyang, Liaoning, China \\ ${ }^{4}$ Department of Anesthesia, West China Second Hospital, \\ Sichuan University, Chengdu, Sichuan, China \\ Corresponding authors: D. Luo / X.D. Kong \\ E-mail: genendisease@163.com / kongxd@263.net
}

Genet. Mol. Res. 13 (4): 8234-8240 (2014)

Received August 14, 2014

Accepted September 27, 2014

Published October 8, 2014

DOI http://dx.doi.org/10.4238/2014.October.8.5

\begin{abstract}
Isolated methylmalonic acidemia (MMA) is a genetically heterogeneous disorder caused mainly by deficiency of methylmalonyl-CoA mutase. In the present study, we analyzed MUT gene mutations in 3 Chinese couples with a birth history of isolated MMA. We also provided prenatal diagnoses for the detected mutation. Exons and exon-intron boundaries of the MUT gene were analyzed by polymerase chain reaction and direct sequencing. Prenatal genetic diagnoses were performed by chorionic villus sampling after the genotypes of parents were determined. Six heterozygous mutations in the MUT gene were identified in the 3 families, including c. $1880 \mathrm{~A}>\mathrm{G}$ (p.H627R) and IVS9-1G $>$ A for family 1, c.1741C $>$ T (p.R581X) and
\end{abstract}


c.729insTT (p.D244fX39) for family 2, and c.616C $>$ T (p.Q206X) and c.1280G $>$ A (p.G427D) for family 3. Among these, c.616C $>$ T (p.Q206X), c.1280G $>$ A (p.G427D), IVS9-1G $>$ A, and c.1741C $>$ T (p.R581X) were novel mutations. These mutations were not detected in 100 normal controls. The fetus in pedigree 3 was free of the mutations carried by the parents, while the fetuses in pedigrees 1 and 2 were heterozygous mutation carriers. All 3 families decided to continue with their pregnancies and the neonates did not show any symptoms of MMA after birth. Our results indicated that mutations in the MUT gene are the primary cause of isolated MMA, and that most mutations were novel. For families with early-onset isolated MMA, direct sequencing of the $M U T$ gene is crucial for genetic counseling, prenatal diagnosis, and identification of carriers.

Key words: Isolated methylmalonic aciduria; $M U T$ gene; Mutation; Prenatal diagnosis

\section{INTRODUCTION}

Methylmalonic aciduria (MMA) includes a group of autosomal recessive genetic diseases that occur primarily due to a deficiency in methyl malonyl coenzyme A mutase (MCM) or metabolic defects of its coenzyme, glandglycosides cobalamin (Ado-Cbl) (Fowler et al., 2008; Tang et al., 2009). Based on their biochemical features, affected children may be classified as simple MMA or MMA combined with homocysteine hyperlipidemia. The gene encoding MCM, MUT (MIM 251000), has been mapped to chromosomal region 6p21.2-p12 (Jansen et al., 1989), which consists of 13 exons and encodes for 750 amino acids. Mutations in the MUT gene have been shown to be capable of causing simple MMA. Detection of MUT gene mutations can provide crucial clues for diagnosing MMA. However, its application for prenatal diagnosis has not been described. We report the diagnosis of 3 families with a birth history of simple MMA through genetic diagnoses, for whom prenatal diagnosis was achieved during early gestation.

\section{MATERIAL AND METHODS}

\section{Patients}

Three couples who had previously given birth to children affected with MMA came to our prenatal diagnosis center between 2009 and 2011 for genetic counseling. Probands of the 3 families, 1 male and 2 females (Figure 1), were all full-term born and manifested symptoms within 1 month, with the main features including repeated vomiting, lethargy, convulsions, anorexia, delayed mental and physical developmental or retrogression, hypotonia, abnormal behavior, and psychiatric symptoms. Gas chromatograph-mass spectrometry (GC-MS) analysis of urinary organic acid (Metabolic Disease Laboratory, Beijing Pediatric Hospital or MILS Laboratory of Inborn Errors of Metabolism of Japan) indicated the concentration of urinary methyl malonic acid to be significantly higher than normal. For the 3 patients, respectively, these values were measured to be $76.7,336.2$, and $176.3 \mathrm{mM}$ (reference value 0.2-3.6 mM). 
In addition, methyl citrate was slightly elevated $(2.90,4.13$, and $1.53 \mathrm{mM}$ for the 3 patients, respectively; reference range $0-1.1 \mathrm{mM}$ ). Measurement of serum homocysteine was normal. A vitamin $B_{12}$ load test for all 3 children was invalid, which was in line with the diagnosis of simple MMA. The probands all perished within 6 months. In one family, the elder sister of the proband (also perished) showed the same symptoms during the neonatal period.

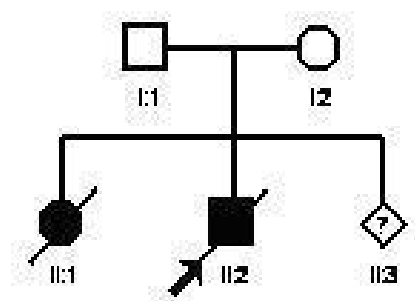

Family 1

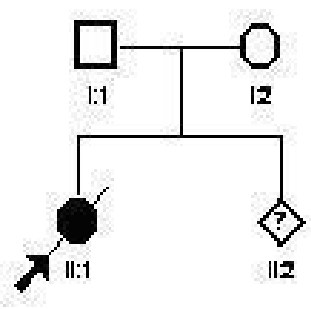

Family 2

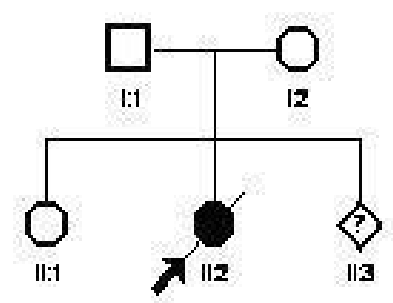

Family 3

Figure 1. Pedigrees of the three families affected with methylmalonic aciduria. The probands are indicated with an arrow.

\section{DNA extraction}

After obtaining informed consent, peripheral venous blood samples were obtained from the 3 couples. DNA was extracted from $2 \mathrm{~mL}$ ethylenediaminetetraacetic acid (EDTA) anti-coagulated blood. During the 11th gestational week, chorionic villus samples were obtained under the guidance of trans-abdominal ultrasound. Fetal DNA was extracted with a kit produced by Tiangen Biotech (Beijing, China) following manufacturer instructions.

\section{Polymerase chain reaction (PCR) and DNA sequencing}

Fourteen pairs of PCR primers specific to the MUT gene's 13 exons, exon-intron junction regions, and part of the promoter region were synthesized based on the literature (Wang et al., 2009). The $M U T$ gene was subjected to PCR amplification in a $25-\mu \mathrm{L}$ reaction volume. The reaction conditions have been described by Wang et al. (2009). PCR products were purified using an ABI BigDye3.1 Sequencing Kit, and were sequenced in both directions using an ABI3130xl gene sequencer. Sequencing results were compared with the normal sequence. Potential mutations were identified using the Chromas software.

\section{Nomenclature of mutations and verification}

Sequence variants were initially searched against PubMed (http://www.ncbi.nlm.nih. gov/PubMed) and the MMA database (http://www.genomed.org/lovd). All new mutations were verified against corresponding sequences from 100 healthy unrelated individuals to rule out polymorphisms. New mutations were named according to the "Recommendations for the Description of Sequence Variants" guidelines published by the Human Genome Variation Society (http://www.hgvs.org/mutnomen/recs.html). 


\section{Prenatal diagnosis}

Following detection of $M U T$ gene mutations in the parents, fetal villus samples were obtained at the 11th gestational week for prenatal diagnosis. Following DNA extraction, MUT gene mutations were detected by PCR and DNA sequencing.

\section{Exclusion of maternal contamination}

For prenatal diagnosis, maternal contamination of the choronic villus samples was excluded using a Promega PowerPlex 16 HS System kit (Promega, Fitchburg, WI, USA). The product was subjected to fragment analysis with an ABI3130X1 gene sequencer and the GeneMapper v3.2 software to exclude maternal contamination. The criteria for exclusion of maternal pollution were: fetus allelic fluorescence peaks derived from both parents and no fetus point 2 fluorescence detection peak representative of the mother was found.

\section{Follow-up}

After the births, umbilical cord blood specimens were collected for genetic diagnosis, and neonatal urinary organic acid was screened by GC-MS.

\section{RESULTS}

\section{Detection of $M U T$ gene mutations}

In family 1, the proband's mother carried a heterozygous c.1880A $>\mathrm{G}$ (H627R) mutation, while her father carried a heterozygous IVS9-1G $>$ A mutation. In family 2 , the proband's mother carried a heterozygous c. $1741 \mathrm{C}>\mathrm{T}$ (R581X) mutation, and the father carried a heterozygous c.729insTT (D244fX39) mutation. In family 3, the proband's mother carried a heterozygous c.616C $>\mathrm{T}(\mathrm{Q} 206 \mathrm{X})$ mutation, while the father carried a heterozygous c.1280G $>\mathrm{A}$ (G427D) mutation (Figure 2). Among these mutations, p.Q206X IVS9-1G $>$ A and p.R581X, p.H627R have not been described previously. Sequencing of the corresponding region in the 100 healthy individuals showed none of the above mutations except for 2 known single nucleotide polymorphisms, including c.636G $>$ A K112K and c.1595G $>$ A (R532H).

\section{Results of prenatal diagnosis}

For family 1, the fetus was found to have the heterozygous H627R mutation, but no IVS9-1G $>$ A mutation. For family 2 , the fetus carried a heterozygous R581X mutation, but no D244fX39 mutation. For family 3 , the fetus did not carry either of the same mutations as the parents (Figure 2).

\section{Exclusion of maternal contamination}

As verified using a PowerPlex 16-HS system kit, none of the chorionic villus samples showed maternal contamination. 


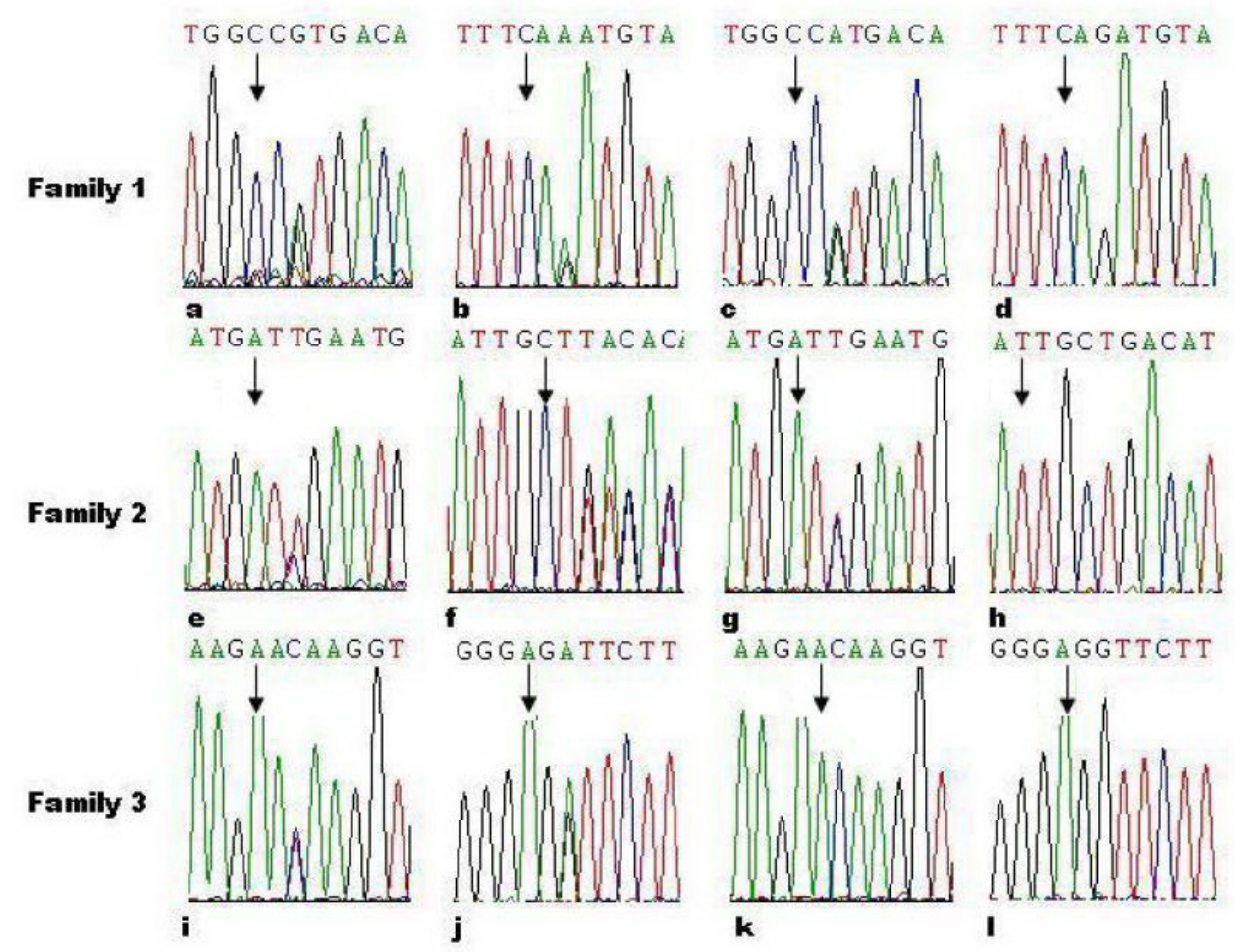

Figure 2. Sequencing results of the three families with a birth history of methylmalonic aciduria. For family 1 , the proband's mother has carried a heterozygous p.H627R mutation (a), and the father has carried a heterozygous IVS9-1G $>$ A mutation (b). The fetus was found to carry a heterozygous p.H627R mutation (c) but no IVS9-1G $>$ A mutation (d). For family 2, the proband's mother has carried a heterozygous p.R581X mutation (e), while the father has carried a heterozygous p.D244fX39 mutation (f). The fetus was found to carry a heterozygous p.R581X mutation (g) but no p.D244fX39 mutation (h). In family 3, the proband's mother has carried a heterozygous p.Q206X mutation (i), the father has carried a heterozygous p.G247D mutation (j). The fetus did not carry either the p.Q206X (k) or p.G247D mutation (I).

\section{Follow-up}

The fetuses of families 1 and 2 carried heterozygous p.H627R and p.R581X mutations, respectively, and they likely had a normal phenotype after birth. Since the fetus of family 3 did not carry the same mutation of the parents, the risk for developing the disease was even lower. After genetic counseling, all 3 families decided to continue with their pregnancies. The fetuses were born at full-term. Umbilical cord blood was sampled for genetic diagnoses, and the results were consistent with those of prenatal diagnoses. Neonatal urine screening was normal. All fetuses were followed-up by telephone for 1 year, and all reported normal general development.

\section{DISCUSSION}

Wild-type MCM comprises a polypeptide chain of 750 amino acids with an initial sequence of 32 amino acids, which is truncated after entering the mitochondria. The remain- 
ing chain is assembled into an isodimer, which can bind with 2 Ado-Cbl molecules to form the fully functional enzyme. Each subunit of MCM has two major functional domains, a functional domain located at the N-terminal end, which includes a structure connecting the 2 subunits (amino acids 33-87) and a substrate-binding region (amino acids 88-422). The second functional domain is located within the binding domain of the C-terminal of the Ado-Cbl coenzyme (amino acids 578-750). In addition, the substrate-binding domain and the coenzyme-binding domain are connected by a long segment (amino acids 423-577). Most MUT gene mutations identified to date have been shown to affect the structure of the substrate- or coenzyme-binding region for the MCM substrate (Nham et al., 1990; Mancia et al., 1999).

By December 2012, 242 MUT gene mutations had been described (Human Gene Mutation Database HGMD $^{\circledR}$ Professional 2012.4 version), and mostly included nonsense and missense mutations. Splice site mutations, deletions, and insertions are relatively rare (Acquaviva et al., 2005; Worgan et al., 2006; Dündar et al., 2012). Most mutations have been found only once and are restricted to a single family, and there have been no mutation hotspots identified. In China, Wang et al. (2009) found 17 mutations in 14 MMA patients, but without a mutation hotspot; the mutation c.1630-1631GG>TA (p.G544X) is the most common, with a frequency of $14.3 \%$. In the present study, we identified 6 mutations among 3 couples. Among these, p.H627R and p.D244fX39 were found previously, which, respectively, affect the N-side substrate binding region and the C-terminal coenzyme-binding region. Mutations p.Q206X, p.G427D, IVS9-1G>A, and p.R581X were novel mutations. Because these mutations were not observed in the 100 healthy controls, they were speculated to be pathogenic. The p.Q206X and p.R581X nonsense mutations resided, respectively, in exons 3 and 10, which can give rise to truncated peptide chains, affecting the substrate-binding domain and the coenzyme-binding domain, respectively. The p.G427D mutation resides in the connection domain, although its influence on mutase is unclear. IVS9-1G $>$ A results in deletion of exon 10 or a splicing error.

Children with MMA caused by an MCM defect often manifest a serious condition with acute onset within a few hours to several days after birth. Early symptoms may be nonspecific, but the condition rapidly progress, featuring a high mortality rate during the neonatal period and a poor prognosis (Dündar et al., 2012; Liu et al., 2012). Birth of affected children can be prevented by prenatal diagnosis. By determining MMA in amniotic fluid or maternal urine, activity of mutase in cultured amniocytes or chorionic villus cells, or metabolites of cobalamins of amniotic fluid cells, prenatal diagnosis for this disease can be accomplished (Zhang et al., 2008; Inoue and Ohse, 2011). Recently, tandem mass spectrometry analysis of peptidyl carnitine has been used for prenatal diagnosis. MUT mutation screening for affected children by DNA sequencing and prenatal genetic diagnosis for subsequent pregnancies can prevent the birth of affected children. In the present study, we performed prenatal diagnoses for 3 families with a reproductive history of MMA using chorionic villus samples. Based on the results of prenatal diagnosis, we predicted that 3 fetuses would be normal. The results were confirmed after the babies were born. Urine screening and developmental parameters were all normal, confirming that the $M U T$ gene diagnosis is an effective means for prenatal diagnosis.

\section{ACKNOWLEDGMENTS}

Research supported by the Fundamental Research Fund for Central Universities (\#N110804002) and a Support Program from the Department of Science and Technology of Sichuan Province (\#2012SZ0027). 


\section{REFERENCES}

Acquaviva C, Benoist JF, Pereira S, Callebaut I, et al. (2005). Molecular basis of methylmalonyl-CoA mutase apoenzyme defect in 40 European patients affected by mut(o) and mut- forms of methylmalonic acidemia: identification of 29 novel mutations in the MUT gene. Hum. Mutat. 25: 167-176.

Dündar H, Ozgul RK, Guzel-Ozanturk A, Dursun A, et al. (2012). Microarray based mutational analysis of patients with methylmalonic acidemia: identification of 10 novel mutations. Mol. Genet. Metabol. 106: 419-423.

Fowler B, Leonard JV and Baumgartner MR (2008). Causes of and diagnostic approach to methylmalonic acidurias. $J$. Inherit. Metab. Dis. 31: 350-360.

Inoue Y and Ohse M (2011). Prenatal diagnosis of methylmalonic aciduria by measuring methylmalonic acid in dried amniotic fluid on filter paper using gas chromatography-mass spectrometry. Anal. Bioanal. Chem. 400: 1953-1958.

Jansen R, Kalousek F, Fenton WA, Rosenberg LE, et al. (1989). Cloning of full-length methylmalonyl-CoA mutase from a cDNA library using the polymerase chain reaction. Genomics 4: 198-205.

Liu YP, Ma YY, Wu TF, Wang Q, et al. (2012). Abnormal findings during newborn period of 160 patients with early-onset methylmalonic aciduria. Zhonghua Er. Ke Za Zhi 50: 410-414.

Mancia F, Smith GA and Evans PR (1999). Crystal structure of substrate complexes of methylmalonyl-CoA mutase. Biochemistry 38: 7999-8005.

Nham SU, Wilkemeyer MF and Ledley FD (1990). Structure of the human methylmalonyl-CoA mutase (MUT) locus. Genomics 8: 710-716.

Tang H, Hao H, Tang SH, Chen X, et al. (2009). Mutation analysis of the MMACHC gene in a pedigree with methylmalonic aciduria. Zhonghua Yi Xue Yi Chuan Xue Za Zhi 26: 62-65.

Wang F, Han L, Ye J, Qiu W, et al. (2009). Analysis of the MUT gene mutations in patients with methylmalonic acidemia. Zhonghua Yi Xue Yi Chuan Xue Za Zhi 26: 485-489.

Worgan LC, Niles K, Tirone JC, Hofmann A, et al. (2006). Spectrum of mutations in mut methylmalonic acidemia and identification of a common Hispanic mutation and haplotype. Hum. Mutat. 27: 31-43.

Zhang Y, Yang YL, Hasegawa Y, Yamaguchi S, et al. (2008). Prenatal diagnosis of methylmalonic aciduria by analysis of organic acids and total homocysteine in amniotic fluid. Chin. Med. J. 121: 216-219. 